\title{
The Geometry of the Excited Charge Transfer States: Flattening or Twisting?
}

\author{
J. Dobkowski And I. SAZANovich* \\ Institute of Physical Chemistry, Polish Academy of Sciences \\ Kasprzaka 44, 01-224 Warsaw, Poland \\ Dedicated to the memory of Professor Jerzy Prochorow
}

\begin{abstract}
Numerous molecules built of an electron acceptor and electron donor units linked together by single bond exhibit a peculiar behavior in their low-lying electronically excited states. N,N-diethylaminoacetophenone and 4-acetyl-4'-dimethylamino-biphenyl, having acetophenone as the acceptor and differentiated by the donors groups $\mathrm{N}\left(\mathrm{CH}_{3}\right)_{2}$ and dimethylaniline, respectively, were selected as the subject of study. To recognize the excited state relaxation paths the stationary and time-resolved spectroscopy in absorption and emission was applied. Experimental results indicate that after excitation in polar solvents $\mathrm{N}, \mathrm{N}$-diethylaminoacetophenone reduces to minimum the overlap between $\pi$-electron systems of the donor and acceptor groups relaxing to the twisted internal charge-transfer state; contrary to that, flattening of the 4-acetyl-4'-dimethylamino-biphenyl skeleton generates increase in the overlap of the $\pi$-electron systems.
\end{abstract}

PACS numbers: 33.50.--j, 61.66.Hq

\section{Introduction}

The excited states characterized by significant or full transfer of electron from a donor to an acceptor group can be categorized with application of the minimum/maximum overlap rule $[1,2]$. This rule postulates that the overlap between the donor and acceptor orbitals involved in the electron transfer should reach minimum or maximum value. The increase in the orbital overlap generated by flattening of the molecular skeleton in excited charge-transfer (CT) states was established in the case of anthryl [3], acridine [4], and pyrene [5] derivatives of $\mathrm{N}, \mathrm{N}$-dimethylaniline. The minimum overlap can be achieved by:

*Permanent address: Institute of Molecular and Atomic Physics, Academy of Sciences of Belarus, F. Scaryna Avenue 70, 220072 Minsk, Belarus. 
(i) location of the node of the orbital on one of the atoms creating a single bond between donor and acceptor group [6-9];

(ii) significant geometry transformation of the molecular skeleton, which leads to the donor and acceptor orbital decoupling (twisted internal charge-transfer (TICT) model [10]).

According to the TICT model the emission occurs from two different excited states. The short-wavelength fluorescence $\left(F_{b}\right)$ is emitted from the state characterized by planar geometry, similar to that of the ground state. The long-wavelength $\left(F_{a}\right)$ fluorescence originates from the state denoted by $a^{*}$ (TICT). This state has the donor (D) and acceptor (A) twisted by $90^{\circ}$ with respect to each other. A full one-electron transfer occurs from the donor to acceptor (Fig. 1). The TICT state can be approximated by the structure of a bonded radical pair $\mathrm{D}^{+}-\mathrm{A}^{-}[11]$.

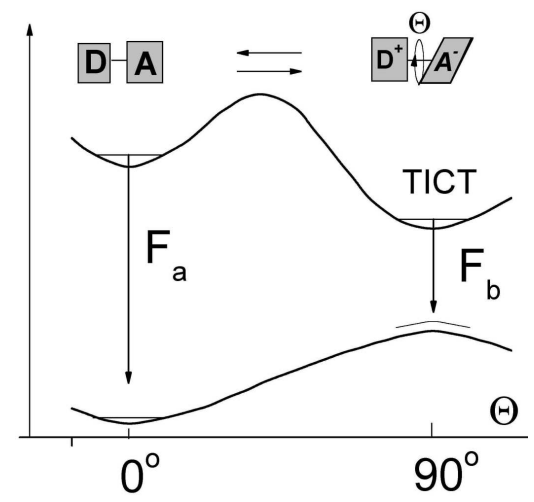

Fig. 1. TICT model. Schematic cross-section of the ground state and lowest excited singlet state potential hypersurfaces along the reaction coordinate represented by the twist angle $\Theta$.

Since with the formation of the charge transfer state a significant increase in the dipole moment took place, the solvent stabilization plays important role on the relaxation path [12-14].

In this paper we compare the excited state relaxation processes in the case of 4-acetyl-4'-N,N-dimethylaminobiphenyl (ADAB) and N,N-diethylaminoacetophenone (DEAA) molecules possessing the donor groups either with the extended $\pi$-electron system (N,N-dimethylaniline) or with a compact $\pi$-electron system localized on the nitrogen atom of the N,N-dialkylamino group, respectively (Scheme 1). Both molecules have identical acceptors (acetophenone). For the sake of understanding of the excited state geometry transformation the two compounds, 3-methyl-N,N-dimethylamino-benzaldehyde (mDMADA) and 4-acetyl$-2,2^{\prime}$-dimethyl-4'-N,N-dimethylaminobiphenyl (ADAB-Me) were synthesized. In both model compounds the donor units are pretwisted with respect to the acceptor chromophore by the steric effect of the methyl groups. 
<smiles>CCCCCCCCCCCCCCCCCCN(C)c1ccc(C)cc1C</smiles><smiles>CCN(CC)c1ccc(C(C)=O)cc1</smiles>
DEAA<smiles>CC(=O)c1ccc(-c2ccc(N(C)C)cc2)cc1</smiles>

$A D A B$<smiles>CC(=O)c1ccc(-c2ccc(N(C)C)cc2C)c(C)c1</smiles>

ADAB-Me

Scheme 1

The goal of this work is elucidation of the excited state relaxation paths in the case of DEAA and ADAB. The time-resolved spectroscopy in absorption and emission was applied for monitoring the excited state electron transfer associated with geometry transformation. One of the aims was to understand the role of the solvent cage reorganization on the excited state energy degradation.

\section{Experimental}

$\mathrm{ADAB}$ was synthesized as described previously [15]. $\mathrm{ADAB}^{-\mathrm{CH}_{3}}$ was synthesized in a 4-step procedure. (1) Commercial 3-methyl-4-bromo-acetophenone was heated in nitrobenzene with 2-iodotoluene and catalytic $\mathrm{Cu}$. (2) The resulting 4-acetyl-2,2'-dimethyl-biphenyl was nitrated by $\mathrm{HNO}_{3}$ to 4-acetyl-2,2'-dimethyl-4'-nitro-biphenyl; (3) the latter was catalytically reduced $\left(\mathrm{H}_{2} / \mathrm{PtO}_{2}\right)$ to 4-acetyl-2,2'-dimethyl-4'-amino-biphenyl, and methylated with $\mathrm{CH}_{3} \mathrm{I}$ to the desired 4-acetyl-2,2'-dimethyl-4'-N,N-dimethylamino-biphenyl (ADAB-Me). DEAA was obtained by acetylation of diethylaniline, applying the method of Kosolapoff [16a]. The product was purified by column chromatography. mDMABA was synthesized in accordance with the procedure described in [16b].

Solvents (Merck, spectral grade): cyclohexane (CHX), diethylether (ether), acetonitrile (ACN), dimethyl sulfoxide (DMSO) were used without further purification. Stationary absorption spectra were recorded with a Shimadzu UV 3100 spectrometer. Stationary fluorescence spectra were measured using the Jasny spectrofluorimeter [17] or the FS900 Edinburgh Instrument. The spectra were corrected for the instrumental response using the fluorescence standards. Fluorescence quantum yields were determined using quinine sulfate in $0.05 \mathrm{M} \mathrm{H}_{2} \mathrm{SO}_{4}$ [18] as a standard $(\varphi=0.51)$. The fluorescence decays in nanosecond domain were recorded with the single photon counting unit (Edinburgh Instrument). For recording the transient absorption spectra a home-built picosecond spectrometer was used. Briefly, pulses of $1.5 \mathrm{ps}$ duration $(1055 \mathrm{~nm}$ ) and energy of $4 \mathrm{~mJ}$, with repetition of $33 \mathrm{~Hz}$ were provided by a Light Conversion (Vilnius, Lithuania) Nd:glass laser. The pulses were split into two channels. The first beam after 
conversion into the third harmonic $(352 \mathrm{~nm}$, about $1 \mathrm{~mJ})$ was used for excitation. The second one was used for generation of the picosecond continuum (probing beam). A home-built polychromator, equipped with a Hamamatsu back-thinned, one stage TE-cooled CCD element (S7031-1007) was used as the detection system. The temporal resolution of the spectrometer was $2.5 \mathrm{ps}$. The time-resolved fluorescence (TRF) spectra were recorded by means of a home-made picosecond spectrofluorimeter described in detail elsewhere [19]. Briefly, the first beam was used for excitation $(352 \mathrm{~nm})$. The second beam passed through an optical Kerr shutter and opened it. The fluorescence could be transmitted by the shutter only for the time period in which the opening pulse penetrated the Kerr medium. The opening pulse was delayed with respect to the excitation one by an optical delay line (maximum delay $3000 \mathrm{ps}, 0.1 \mathrm{ps} / \mathrm{step}$ ). The delay time was calculated with respect to the maximum of the excitation pulse. The fluorescence was transmitted to the detection system by a quartz fiber. The detection system consisted of a polychromator (Action SpectraPro-275), and a CCD detector (Princeton Instruments Inc.). The temporal resolution of the spectrofluorimeter was $6.5 \mathrm{ps}$. The spectra were not corrected for the instrumental response.

\section{Results}

\subsection{Stationary spectra}

Room temperature stationary absorption and emission spectra of DEAA and mDMABA recorded in ACN are shown in Fig. 2 (Table I).

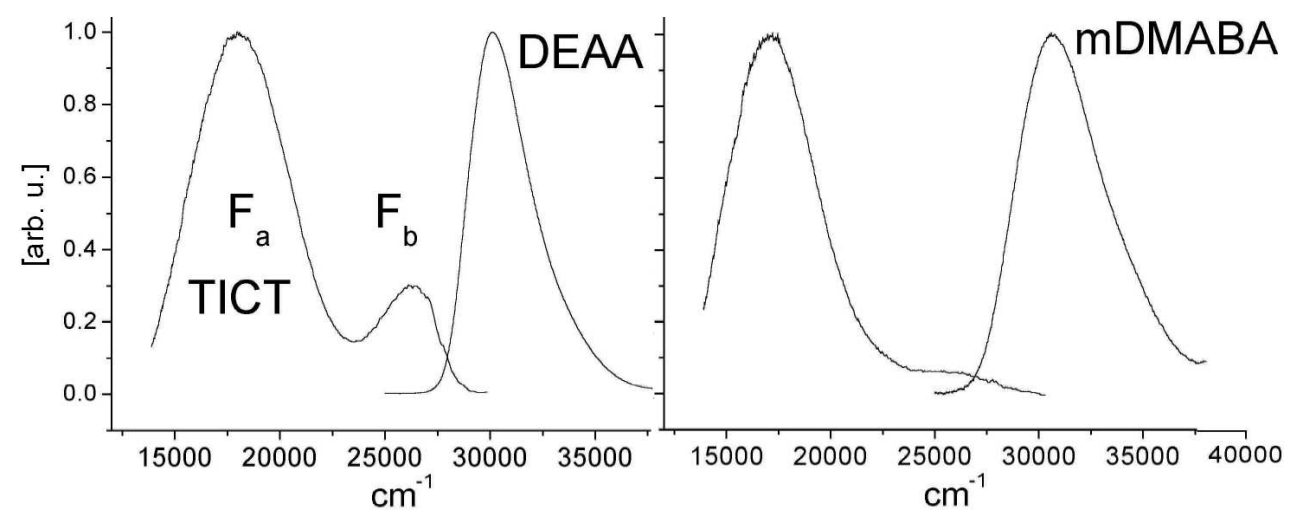

Fig. 2. Room temperature stationary absorption and fluorescence spectra of DEAA and mDMABA in ACN.

The fluorescence spectrum of DEAA consists of two bands located at $26400 \mathrm{~cm}^{-1}\left(F_{b}\right)$ and $18000 \mathrm{~cm}^{-1}\left(F_{a}\right)$. Contrary to that the emission spectrum of mDMABA consists of the low energy band only with the maximum at $17000 \mathrm{~cm}^{-1}$ (Fig. 2, Table I). The shoulder at about $26000 \mathrm{~cm}^{-1}$ is also observed.

Room temperature stationary absorption and emission spectra of ADAB are presented in Fig. 3. 
TABLE I

Absorption and fluorescence maxima, total quantum yields $\left(\varphi_{\text {TOT }}\right)$, the ratio of the quantum yields of the short- and long-wavelength fluorescence $\left(\varphi_{a} / \varphi_{b}\right)$ of DEAA and mDMABA in $\mathrm{ACN}$ at $293 \mathrm{~K}$.

\begin{tabular}{l|c|c|c|c|c}
\hline \hline & Abs. $\max$. & $F_{b} \max$ & $F_{a} \max$ & $\varphi_{\mathrm{TOT}}{ }^{a}$ & $\left(\varphi_{a} / \varphi_{b}\right)$ \\
\hline DEAA & 30000 & 26400 & 18000 & 0.010 & 4.9 \\
mDMABA & 30600 & $26000 \mathrm{sh}$. & 17000 & 0.005 & 16.8 \\
\hline \\
sh - shoulder \\
${ }^{a}$ error $\pm 15 \%$
\end{tabular}

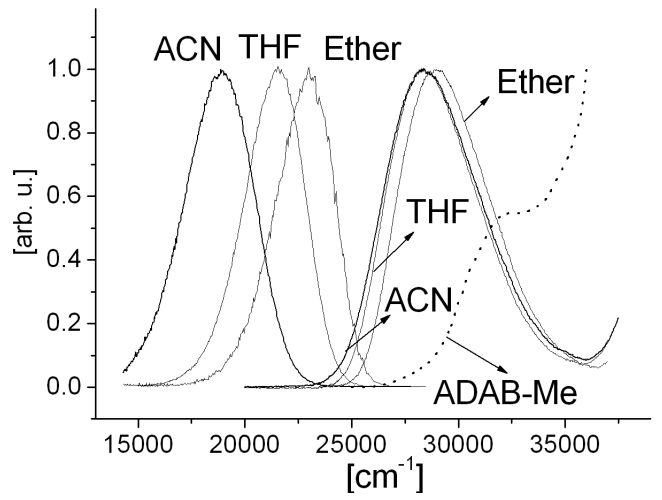

Fig. 3. Room temperature stationary absorption and fluorescence spectra of ADAB in ether, THF and ACN. The dotted curve indicates the room temperature absorption spectrum of ADAB-Me in ACN.

TABLE II

The fluorescence band maxima $\left(\nu_{\max }\left[\mathrm{cm}^{-1}\right]\right)$, quantum yields $\left(\varphi_{\mathrm{F}}\right)$, lifetimes $(\tau[\mathrm{ns}])$, radiative and nonradiative rates $k_{\mathrm{f}}=\varphi_{\mathrm{F}} / \tau, k_{\mathrm{n}}=$ $\tau^{-1}-k_{\mathrm{f}}$ measured for ADAB in solvents characterized by the polarity index $\varepsilon$.

\begin{tabular}{l|c|c|c|c|c|c}
\hline \hline \multicolumn{1}{c|}{ Solvent } & $\varepsilon$ & $\begin{array}{c}\nu_{\max } \\
{\left[\mathrm{cm}^{-1}\right]}\end{array}$ & $\varphi_{\mathrm{F}}$ & $\begin{array}{c}\tau \\
{[\mathrm{ns}]}\end{array}$ & $\begin{array}{c}k_{\mathrm{f}} \\
{\left[10^{9} \mathrm{~s}^{-1}\right]}\end{array}$ & $\begin{array}{c}k_{\mathrm{n}} \\
{\left[10^{9} \mathrm{~s}^{-1}\right]}\end{array}$ \\
\hline diethylether & 4.34 & 23000 & 0.61 & 1.3 & 0.45 & 0.29 \\
terahydrofurane & 7.58 & 21750 & 0.7 & 1.8 & 0.39 & 0.17 \\
acetonitrile & 37.50 & 19150 & 0.36 & 1.9 & 0.19 & 0.34 \\
\hline
\end{tabular}

$\varphi_{\mathrm{F}}$ - error $\pm 10 \%$

${ }^{a}$ measured at the maximum of the band, error: $\pm 0.1 \mathrm{~ns}$.

For $k_{\mathrm{f}}, k_{\mathrm{n}}$, the estimated error was $\pm 15 \%$.

The shape and the maximum of the first absorption band is practically independent of the solvent. The fluorescence consists of one broad band, its spectral position being crucially dependent on the polarity of environment (Table II). The 
maximum of the first absorption band of ADAB-Me $\left(32200 \mathrm{~cm}^{-1}\right)$ is located at considerably higher energy as compared to $\operatorname{ADAB}\left(28300 \mathrm{~cm}^{-1}\right)$, see Fig. 3. The room temperature fluorescence of $\mathrm{ADAB}-\mathrm{Me}$ in $\mathrm{ACN}$ is undetectable.

The absorption of ADAB in ether does not depend significantly on temperature, however the first band shows at low temperatures a small red shift of the maximum, reflecting the changes in the population of the ground state vibrational levels and/or ground state geometry changes (Fig. 4).

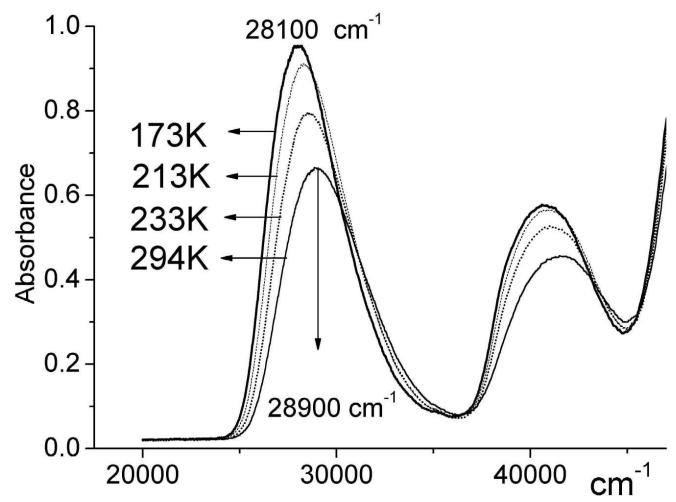

Fig. 4. Low temperature stationary absorption spectra of ADAB in ether.

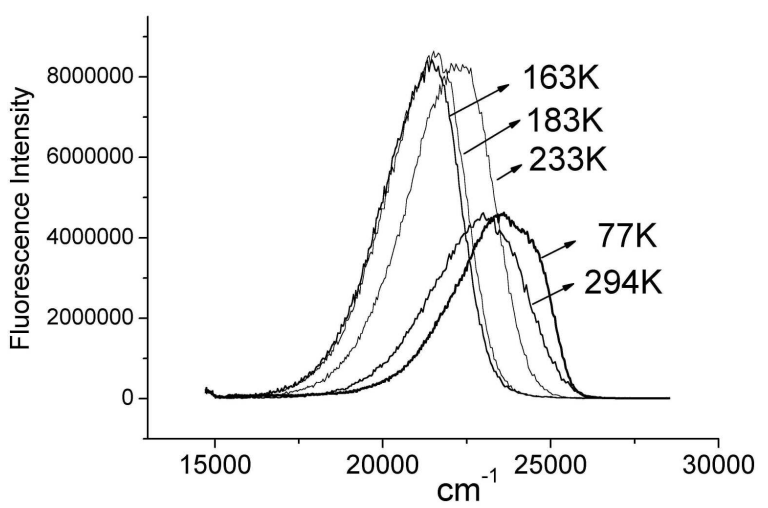

Fig. 5. Stationary fluorescence spectra of ADAB in ether recorded as a function of temperature. Spectrum recorded at $77 \mathrm{~K}$ was normalized to the maximum of the fluorescence spectrum recorded at $294 \mathrm{~K}$.

Contrary to the absorption, the fluorescence spectra exhibit significant temperature dependence (Fig. 5). The maxima of the fluorescence of ADAB recorded in liquid ether at selected monotonically decreasing temperatures reveal red shift, however near the freezing point a reversed tendency is observed. 


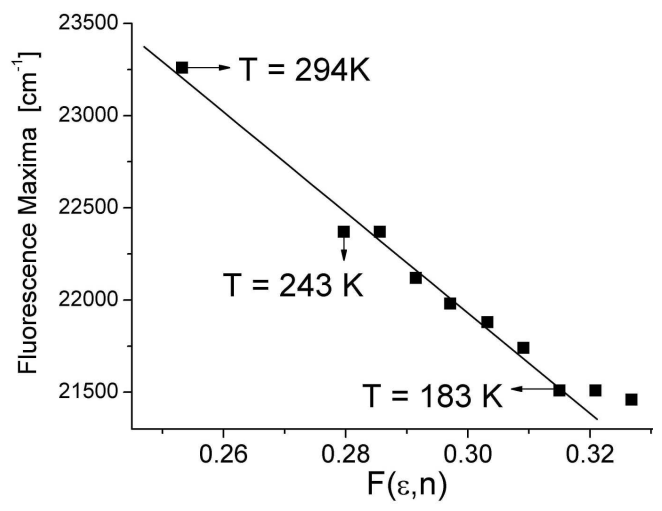

Fig. 6. Plot of thermochromic shift of the fluorescence maxima of ADAB in ether. Two lowest temperature points were not included in the fitting procedure. Values of the $F(\varepsilon, n)$ function were taken from Ref. [20].

The plot of the thermochromic shifts of the fluorescence maxima versus the temperature dependent polarity function $F(\varepsilon, n)$ is shown in Fig. 6

$$
F(\varepsilon, n)=\frac{\varepsilon-1}{2 \varepsilon+1}-\frac{1}{2} \frac{n^{2}-1}{2 n^{2}+1} .
$$

From the thermochromic data (Fig. 6), a value of the excited state dipole moment $\left(\mu_{\mathrm{e}}\right)$ of the emitting singlet state can be calculated using the formula [21, 22]:

$$
\tilde{\nu}_{\max }=-\frac{2 \boldsymbol{\mu}_{\mathrm{e}} \cdot\left(\boldsymbol{\mu}_{\mathrm{e}}-\boldsymbol{\mu}_{\mathrm{g}}\right)}{h c a_{0}^{3}} F(\varepsilon, n)+\text { const. }
$$

The excited state dipole moment $\left(\mu_{\mathrm{e}}\right)$ was determined from the relation (2) and was found as $27 \pm 4 \mathrm{D}$. The values of the ground state dipole moment $\left(\mu_{\mathrm{g}}\right)$ and the Onsager cavity radius $\left(a_{0}\right)$ were assumed to be equal to $5.65 \mathrm{D}$ and $6 \AA$, respectively, as for the cyano analogue of $\mathrm{ADAB}$ [23].

\subsection{Time-resolved experiments}

Room temperature transient absorption (TA) spectra of DEAA in CHX and DMSO recorded for two selected delay times $\left(\tau_{\mathrm{d}}\right)$ are shown in Fig. 7A, B. The difference between the TA spectra recorded in DMSO for the delay of 20 ps and $2850 \mathrm{ps}$ is shown in Fig. 7C and compared with the absorption spectrum of the anion radical of acetophenone [24] as well as with the TA spectrum of the pretwisted model compound.

Room temperature TRF spectra of DEAA in ACN recorded as a function of delay time are shown in Fig. 8A. During the 10 ps period the primary $\left(F_{b}\right)$ emission practically disappears. The intensity of the secondary, long-wavelength fluorescence $\left(F_{a}\right)$ remains constant. The kinetic curve of the rise and decay of the $F_{b}$ emission matches well the kinetic profile of the excitation pulse (Fig. $8 \mathrm{~B}$ ). Because the decay of the $F_{b}$ fluorescence is faster than the resolution of the apparatus, the rise of the long-wavelength emission cannot be properly monitored. The lifetime of $F_{a}$ is infinite on the timescale $\langle 0-50 \mathrm{ps}\rangle$. The fast component observed 

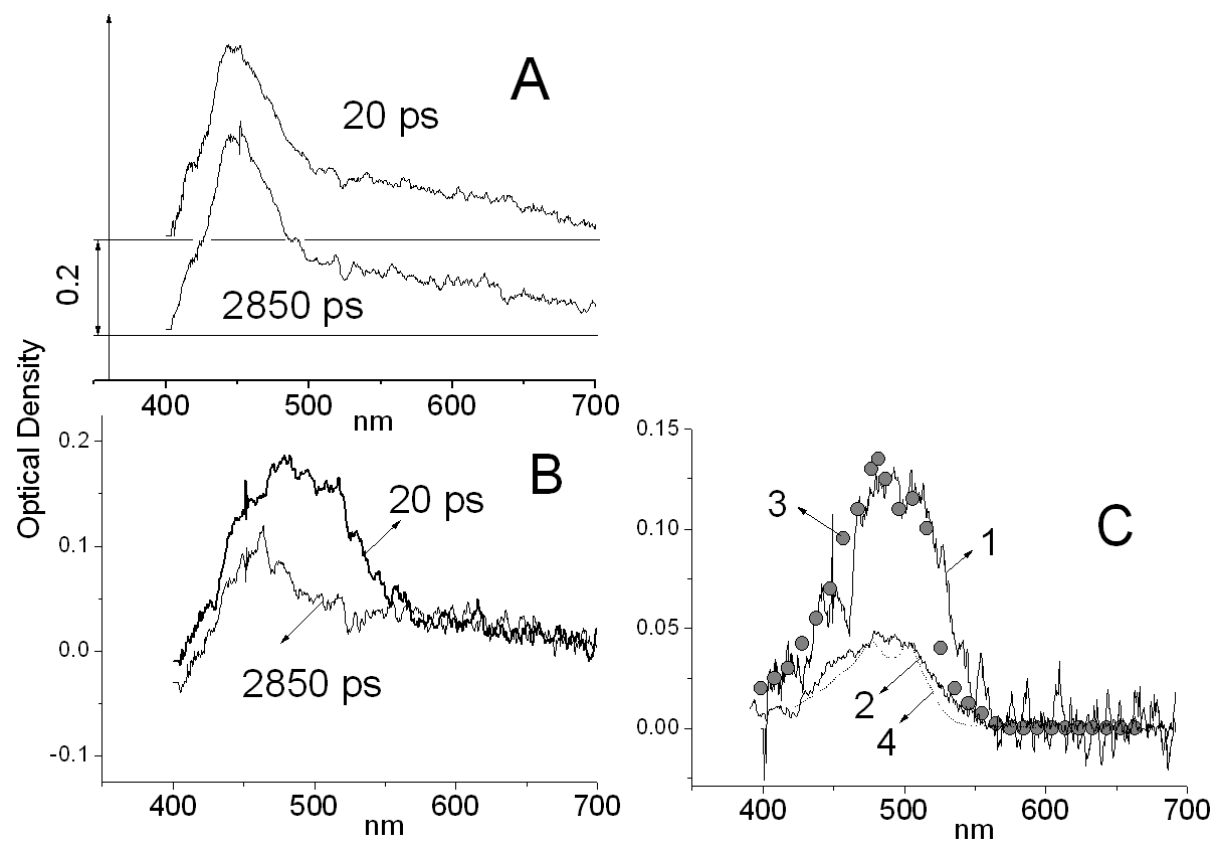

Fig. 7. Room temperature TA spectra of DEAA in cyclohexane (A) and DMSO (B). The spectra presented in $(\mathrm{C})$ are: the spectrum obtained when subtracting the TA spectra for DEAA in DMSO observed with the delay of 20 ps and 2850 ps (1); absorption spectrum of mDMABA in ACN recorded for delay time 42 ps (2); absorption spectrum of radical anion of acetophenone (3) and benzaldehyde (4).
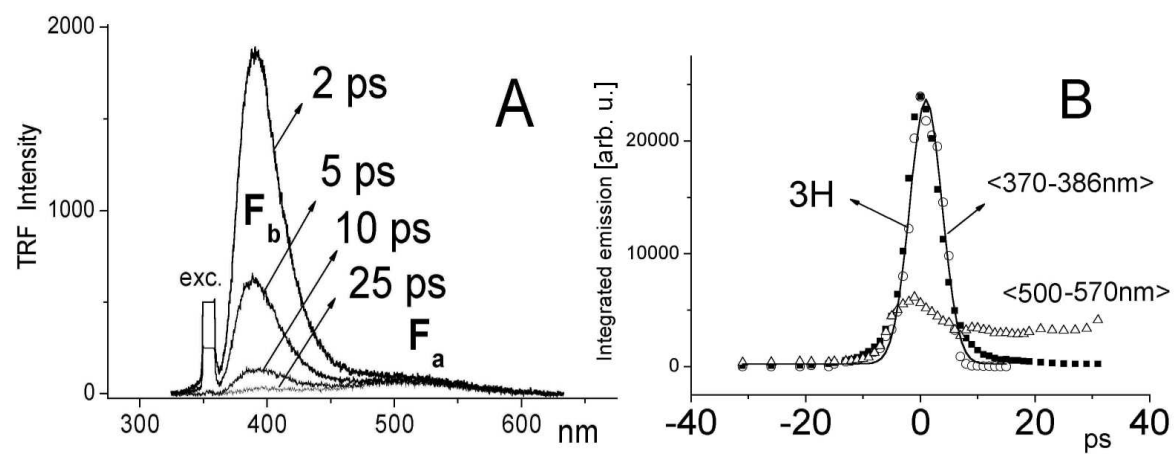

Fig. 8. (A) Room temperature TRF spectra of DEAA in ACN recorded as a function of the delay time. (B) TRF decay curve for the integration range $\langle 370-386 \mathrm{~nm}\rangle$ (squares) and $\langle 500-570 \mathrm{~nm}\rangle$ (triangles); the pulse profile (circles) and its Gaussian fit (solid curve).

in the region of maximum of the excitation pulse is due to the overlap of the tail of the $F_{b}$ band with the $F_{a}$ emission. 
The TRF spectra of ADAB recorded in ether at $294 \mathrm{~K}$ and at $203 \mathrm{~K}$ are shown in Fig. 9. Room temperature TRF spectrum consists of broad band. Just after excitation the maximum of this band is located at $432 \mathrm{~nm}$. For longer delay times the maximum shows the red shift; for example $\lambda_{\max }$ is equal to $440 \mathrm{~nm}$ for $1500 \mathrm{ps}$. The kinetic curve obtained for the blue edge $\langle 390-410 \mathrm{~nm}\rangle$ of the TRF spectrum can be properly approximated by a biexponential decay (Fig. 9A). The decay time obtained for the fast component is $3 \pm 1$ ps and should be treated cautiously, because the resolution of the apparatus is $6 \mathrm{ps}$. This result indicates that temporal evolution of the shape of the spectrum occurs on the scale significantly faster than the fluorescence lifetime of ADAB (Table II).
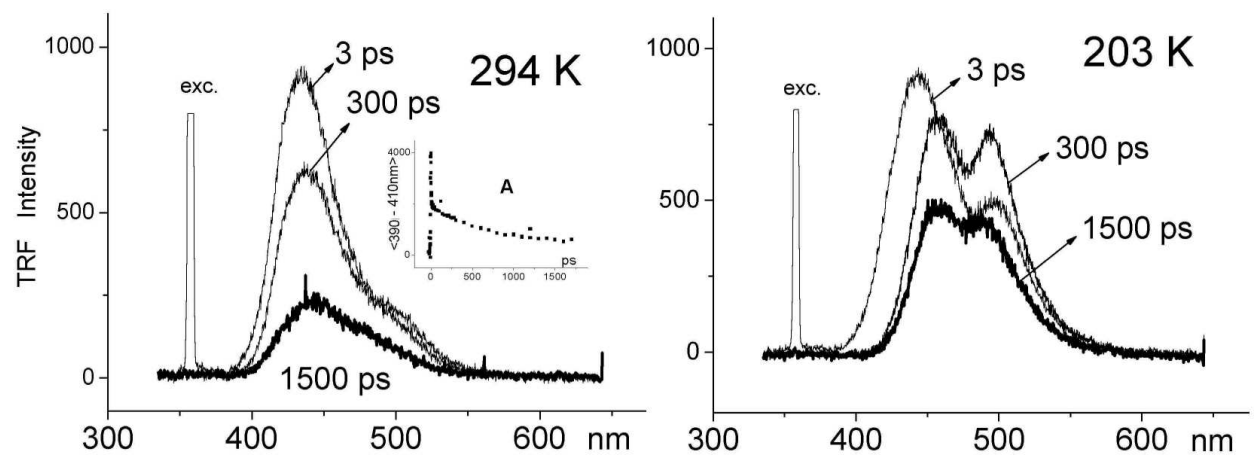

Fig. 9. TRF spectra of ADAB in ether recorded at $294 \mathrm{~K}$ and $203 \mathrm{~K}$ as a function of the delay time. Inset $\mathrm{A}$ - the kinetic curve of the TRF decay obtained for the integration range $\langle 390-410 \mathrm{~nm}\rangle$.

The low temperature TRF spectrum consists of two bands showing an unexpected spectral evolution as a function of delay time. 3 ps after excitation the maximum of the short-wavelength band is located at $445 \mathrm{~nm}$ and the long-wavelength maximum is observed at $498 \mathrm{~nm}$. The spectral position of the short-wavelength maximum depends strongly on the delay time (463 nm for $300 \mathrm{ps}$ ), contrary to that, the long-wavelength maximum exhibits the insignificant blue shift (about $8 \mathrm{~nm})$.

The temporal transformation of the spectral distribution of the short-wavelength TRF band of ADAB in ether was analyzed using the empirical solvation dynamics function, $c(t)$ [12] (Fig. 10). The $c(t)$ function is defined by equation $c(t)=(\tilde{\nu}(t)-\tilde{\nu}(\infty)) /(\tilde{\nu}(0)-\tilde{\nu}(\infty))$, where $\tilde{\nu}(0), \tilde{\nu}(t), \tilde{\nu}(\infty)$ represent the spectral position of the maximum of the fluorescence spectrum just after excitation, at some time after excitation, and at time sufficiently long for reaching solute-solvent equilibration, respectively. The calculated relaxation times $\left(\tau_{\mathrm{s}}\right)$ are collected in Table III.

To recognize the time-dependent evolution of the low temperature TRF spectra of $\mathrm{ADAB}$ in ether the kinetic curves were plotted for $10 \mathrm{~nm}$ intervals starting 


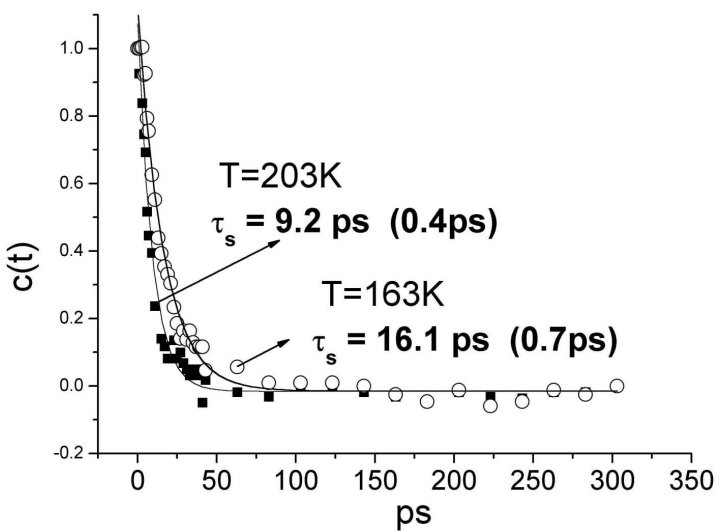

Fig. 10. The $c(t)$ function plot (squares and circles) determined from the TRF maxima of ADAB in ether at $203 \mathrm{~K}$ and $163 \mathrm{~K}$. The solid curves show the best fits.

TABLE III

The relaxation times $\left(\tau_{\mathrm{s}}\right)$ calculated from the $c(t)$ function determined from TRF maxima of $\mathrm{ADAB}$ in ether.

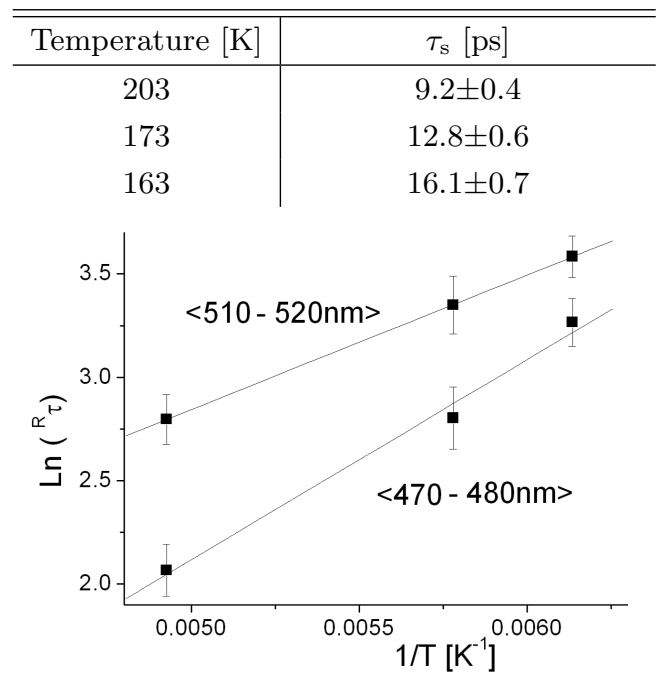

Fig. 11. Plot of the $\ln \left({ }^{\mathrm{R}} \tau\right)$ vs. $1 / T$ for $\mathrm{ADAB}$ in ether. Lines are the linear fits.

from $400 \mathrm{~nm}$ up to $520 \mathrm{~nm}$ and $30 \mathrm{~nm}$ interval $\langle 520-550 \mathrm{~nm}\rangle$ for the red tail of the spectra (Table IV).

For the data collected in Table IV two empirical rules can be formulated:

- The blue part of the emission (400-450 nm) consists of the mono- or two-component decays. The calculated decay times of the fast component increase with $\lambda_{\text {obs }}$. 


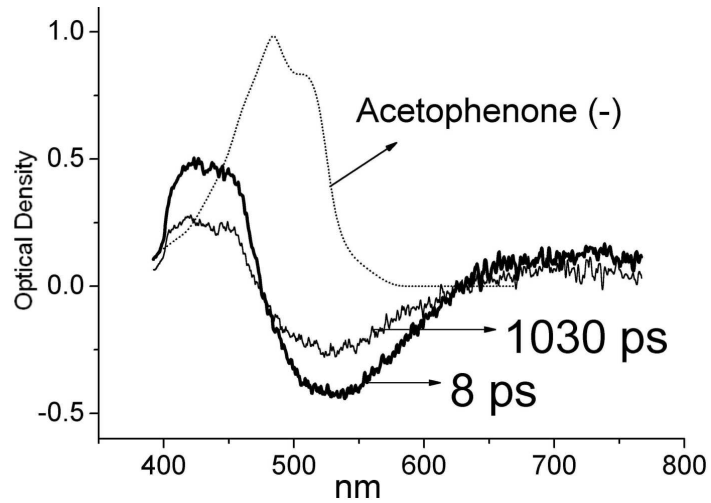

Fig. 12. Room temperature TA spectra of ADAB in ACN recorded for delay times 8 ps and 1030 ps. Dotted curve is the absorption of the anion radical of acetophenone.

TABLE IV

Decay times calculated from TRF experiments for ADAB in diethylether at low temperatures. Decay times longer than 700 ps are denoted as infinity.

\begin{tabular}{|c|c|c|c|c|c|c|}
\hline \multirow[b]{2}{*}{$\begin{array}{c}\left\langle\lambda_{\text {obs }}\right\rangle \\
{[\mathrm{nm}]}\end{array}$} & \multicolumn{2}{|l|}{$203 \mathrm{~K}$} & \multicolumn{2}{|l|}{$173 \mathrm{~K}$} & \multicolumn{2}{|l|}{$163 \mathrm{~K}$} \\
\hline & $\begin{array}{c}\tau \\
{[\mathrm{ps}]}\end{array}$ & & $\begin{array}{c}\tau \\
{[\mathrm{ps}]}\end{array}$ & & $\begin{array}{c}\tau \\
{[\mathrm{ps}]}\end{array}$ & \\
\hline $400-410$ & $3 \pm 0.5$ & & $2 \pm 1$ & & $3 \pm 0.5$ & \\
\hline $410-420$ & $4 \pm 0.5$ & & $5 \pm 1$ & & $4 \pm 0.5$ & \\
\hline $420-430$ & $6.5 \pm 0.5$ & & $8 \pm 1$ & & $10 \pm 0.5$ & \\
\hline $430-440$ & $9.5 \pm 1$ & inf. & $14 \pm 1$ & inf. & $19 \pm 1$ & inf. \\
\hline $440-450$ & $17.5 \pm 2.5$ & inf. & $25 \pm 2$ & inf. & $43 \pm 3$ & inf. \\
\hline $450-460$ & inf. & & ${ }^{R} 5 \pm 3,50 \pm 20$ & inf. & ${ }^{R} 6 \pm 1,65 \pm 26$ & inf. \\
\hline $460-470$ & inf. & & ${ }^{R} 11 \pm 2.5$ & inf. & ${ }^{R} 15.5 \pm 2$ & $\inf$ \\
\hline $470-480$ & ${ }^{R} 8 \pm 1$ & inf. & ${ }^{R} 16.5 \pm 2.5$ & inf. & $R_{26 \pm 3}$ & inf. \\
\hline $480-490$ & ${ }^{R} 15 \pm 2$ & inf. & ${ }^{R} 30 \pm 4$ & inf. & ${ }^{R} 34 \pm 2$ & inf. \\
\hline $490-500$ & ${ }^{R} 17 \pm 2$ & inf. & ${ }^{R} 29 \pm 4$ & inf. & ${ }^{R} 36.5 \pm 2$ & inf. \\
\hline $500-510$ & ${ }^{R} 12.5 \pm 1.5$, & inf. & ${ }^{R} 28 \pm 3$ & inf. & ${ }^{R} 36.5 \pm 2.5$ & inf. \\
\hline $510-520$ & ${ }^{R} 16.5 \pm 2$ & inf. & ${ }^{R} 28.5 \pm 4$, & inf. & ${ }^{R} 36 \pm 2.5$ & inf. \\
\hline $520--550$ & ${ }^{R} 15.5 \pm 2$ & inf. & ${ }^{R} 29.5 \pm 2$ & inf. & ${ }^{R} 41 \pm 2$ & inf. \\
\hline
\end{tabular}

- The red part of the emission exhibits the rise and decay; the rise time depends on the $\lambda_{\text {obs }}$ in the same way as the decay time of the fast component.

Although for ADAB in ether TRF experiments were performed only at three temperatures below $273 \mathrm{~K}$ (Table IV), the effort was done to evaluate the barrier of the excited state process. For the rise times a good linear correlation of the $\ln \left({ }^{\mathrm{R}} \tau\right)$ 
vs. 1/T is observed (Fig. 11). In the case of decay times, due to considerable spread of points, linear correlation is not satisfactorily fulfilled.

The mean value of the barrier is equal to $E_{\mathrm{br}}=540 \pm 40 \mathrm{~cm}^{-1}$.

The room temperature TA spectra recorded for ADAB in ACN are presented in Fig. 12. These spectra consist of the absorption bands located within the spectral regions $400-470 \mathrm{~nm}$ and $650-750 \mathrm{~nm}$ and the stimulated emission with the maximum at $530 \mathrm{~nm}$. No time-dependent evolution of the TA spectra is observed. The transient absorption band cannot be reproduced by the spectrum of the acetophenone radical anion [24].

\section{Discussion}

The sequence of two lowest singlet states in the case of carbonyl derivatives of N,N-dimethyl and diethylaniline (CDA) depends on the solvent polarity. In nonpolar solvents the lowest singlet state is of $n \pi^{*}$ type, but the $\pi \pi^{*}\left(L_{a}\right)$ state is located only few hundreds $\mathrm{cm}^{-1}$ above it. In polar environment the ${ }^{1} \pi \pi^{*}\left(L_{a}\right)$ state, due to its high dipole moment, is stabilized with respect to ${ }^{1} n \pi^{*}$ which induces the inversion of the states [25]. The energy gap between ${ }^{1} \pi \pi^{*} \leftrightarrow{ }^{3} \pi \pi^{*}$ is significantly bigger than between ${ }^{1} n \pi^{*} \leftrightarrow{ }^{3} n \pi^{*}$ states and this fact explains why in the case of CDA the lowest triplet is of $\pi \pi^{*}$ type [25]. For the configuration of the lowest $n \pi^{*}$ singlet and lowest $\pi \pi^{*}$ triplet, in accordance with the Lower and El-Sayed rule [26], the intersystem crossing populates effectively the lowest triplet state and consequently, the intense phosphorescence is observed. Indeed, in nonpolar or weakly polar frozen solvents, CDA emits exclusively or predominantly phosphorescence $[26,27]$.

Transient absorption spectra recorded for DEAA in CHX consist of a single band with the maximum at about $450 \mathrm{~nm}$ (Fig. 7A). This band does not exhibit any temporal evolution within the accessible range of the delay times and was assigned as $T_{1} \rightarrow T_{n}$ transition. Transient absorption spectra of DEAA in DMSO recorded 20 ps after excitation exhibit band with the maximum at 480-510 nm. This band shows temporal evolution, and for delay time $2850 \mathrm{ps}$ its maximum is located at $450 \mathrm{~nm}$, which suggests that it is the $T_{1} \rightarrow T_{n}$ absorption (Fig. 7). The difference between TA spectra recorded $20 \mathrm{ps}$ and $2850 \mathrm{ps}$ after excitation corresponds well with the spectrum of the anion radical of acetophenone. The TA spectrum recorded for mDMABA with the $\mathrm{N}\left(\mathrm{CH}_{3}\right)_{2}$ group pretwisted with respect to the benzene ring matches well the difference spectrum of DEAA as well as the absorption band of the corresponding anion radical of benzaldehyde. The results described above indicate directly that full charge separation takes place after the excitation. Additionally, the $\varphi_{a} / \varphi_{b}$ ratio is 3 times bigger for the pretwisted mDMABA in comparison with planar DEAA molecule (Fig. 2, Table I), which supports the conclusion that in polar solvents the excited state reaction: primary state $\rightarrow$ TICT, plays a leading role on the relaxation path of DEAA.

The crystal structure data obtained for ADAB give the value of the $\Theta$ angle as $33.3^{\circ}[28]$. For ADAB the quantum chemical calculations were not done, how- 
ever the calculations performed for 4-(N,N-dimethylamino)-4'-cyano-biphenyl [29] and for 4-N,N-dimethylanilino-pyrimidine [30] indicate that in the proper analysis of the excited state relaxation path the $\Theta$ angle plays an important role. After excitation, the $\Theta$ angle can increase or decrease. The first case suggests relaxation to the TICT when dimethylaniline and acetophenone subgroups are mutually twisted around the central bond by $90^{\circ}$.

The model compound (ADAB-Me) with the aromatic rings mutually pretwisted in the ground state does not emit fluorescence in polar solvents at room temperature. Having in mind the TICT model (Fig. 1), it is reasonable to assume that after excitation the relaxation of ADAB-Me is dominated by radiationless process: primary excited state $\rightarrow T I C T \rightarrow S_{0}$. In accordance with such hypothesis ADAB in polar solvents should not emit fluorescence, which is not confirmed by the experiment (Fig. 3, Table II). Transient absorption spectra of $\mathrm{ADAB}$ in $\mathrm{ACN}$ do not correspond to the absorption spectrum of acetophenone radical anion (Fig. 12), which indicates that ADAB in excited state cannot be approximated by the structure of bonded radical pair $\mathrm{D}^{+}-\mathrm{A}^{-}$. Summing up: the presented experimental results show that excited state geometry and electronic structure of ADAB cannot be described in terms of the TICT model.

Let us try to analyze the experimental material in terms of relaxation with simultaneous twist of the donor and acceptor subgroups towards a co-planar geometry. The quantum chemical calculations performed for $4^{\prime}$-(pyren-1-yl)benzonitrile [31] and $4^{\prime}$-(pyren-1-yl)acetophenone [32] indicate that the energy of the ${ }^{1} \pi \pi^{*}\left(L_{a}\right)$ state slightly decreases upon flattening of the molecule, and its dipole moment increases. It means that polarity of the solvent induces some flattening of the molecular skeleton (SIF model). In terms of the SIF model the excited state relaxation $S_{0} \rightarrow X \rightarrow Y$, where $X, Y$ denote the primary and secondary excited product, respectively, is barrierless (Fig. 13). Because the low temperature TRF

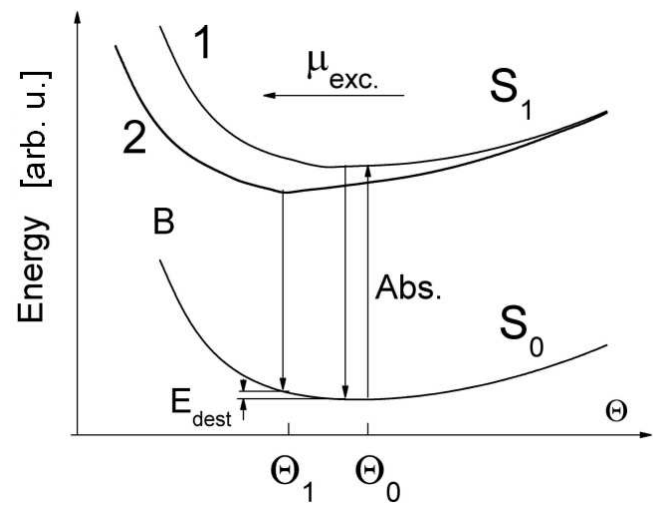

Fig. 13. Proposed energy hypersurface cross-section along the $\Theta$ coordinate. 1 and 2 are modifications of the energy cross-section induced by solvent polarity, $\mu_{\mathrm{exc}}-$ the excited state dipole moment. 

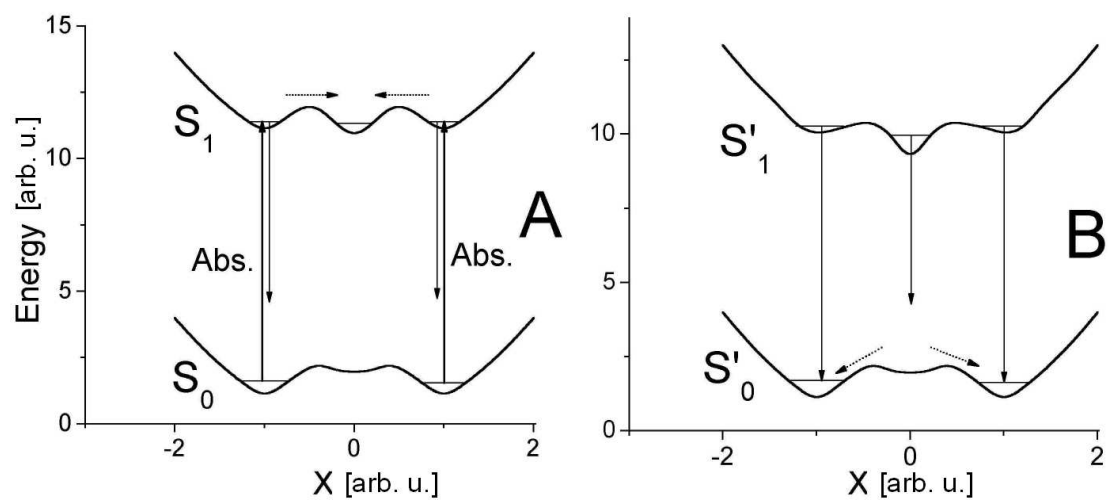

Fig. 14. Proposed energy hypersurface cross-section along the axis $\left\{\Theta_{0}, X\right\}$ (A) and along the axis $\left\{\Theta_{1}, X\right\}(\mathrm{B})$.

spectra of $\mathrm{ADAB}$ in ether exhibit two kinetically coupled fluorescence bands the excited state reaction cannot be barrierless. To solve this problem two coordinate $(\Theta, X)$ relaxation path must be considered. At the beginning we propose (pure speculation) the following properties of the potential hypersurface cross-section along $X$ coordinate:

- the ground state cross-section along the $X$ coordinate exhibits three minima located at 0 and $\pm X_{1}$,

- the cross-section of the excited state shows also a three minima pattern, but the energetic relation between them crucially depends on the solvent polarity. This means that increase in the solvent polarity induces the destabilization of two minima located at $\pm X_{1}$ with respect to the third one located at 0 (Fig. 14A,B).

The barrier, necessary for explanation of the low temperature evolution of the TRF spectra of ADAB in ether, appears along $X$ coordinate. Its height is a function of the angle $\Theta$, and depends on the solvent polarity.

After the excitation the local polarity (solvent cage) depends on time. The reorganization of the solvent cage occurring on the relaxation path stimulates the time-dependent evolution of the potential and consequently generates geometry transformation. The low temperature short-wavelength TRF band of ADAB in ether is emitted along the relaxation path. After termination of the excited state reaction, the fluorescence originated from the equilibrated conformers described by $\left\{\Theta_{1}, 0\right\}$ and $\left\{\Theta_{1}, \pm X_{1}\right\}$.

Few words about the physical meaning of the $X$ coordinate. Because for 4 -N,N-dimethylamino- 4 '-cyanobiphenyl the significant dependence of the fluorescence on the solvent polarity was observed [23], it is reasonable to ascribe the $X$ coordinate to the transformation of the $\mathrm{N}\left(\mathrm{CH}_{3}\right)_{2}$ group. For aniline in gas phase it was experimentally established that in ground state the $\mathrm{NH}_{2}$ group has pyramidal structure, whereas in the first excited state this group is coplanar with the 
aromatic ring [33]. Ground state geometry calculations performed for dimethylaniline more than three decades ago by Dubroca annd Lozano $[34,35]$ indicate that in the ground state the $\mathrm{N}\left(\mathrm{CH}_{3}\right)_{2}$ group is pyramidalized, however a less stable, planar conformer can also exist. Consequently, $X$ can be treated as the wagging coordinate. The excited state correlation between the wagging and $\Theta$ coordinate results from the postulated model. It means that the flattening of the biphenyl rings induces the planar geometry of the dialkylamino group.

There is also another consequence of the proposed scheme: the spectral position of the maxima of the stationary fluorescence recorded in polar solvents depends not only on the solvent stabilization of the $S_{1}$ state but also on the excited state geometry transformation and ground state destabilization (Fig. 13). This can be the reason why the value of the excited state dipole moment calculated from thermochromic shifts is $27 \mathrm{D}$, whereas the calculated value of the excited state dipole moment for 4-N,N-dimethylamino-4'-cyanobiphenyl is only $15.7 \mathrm{D}$ [29].

\section{Acknowledgments}

Authors would like to express the gratitude to Dr Wolfgang Kühnle (Göttingen, Germany) for the synthesis of 4 -acetyl-2,2'-dimethyl-4'-N,Ndimethylaminobiphenyl. One of us (J.D.) thanks Prof. A. Grabowska and Prof. Z.R. Grabowski for the stimulating discussions.

\section{References}

[1] Z.R. Grabowski, J. Dobkowski, Pure Appl. Chem. 55, 245 (1983).

[2] Z.R. Grabowski, K. Rotkiewicz, W. Rettig, Chem. Rev. 105, 3899 (2003).

[3] J. Herbich, A. Kapturkiewicz, Chem. Phys. 170, 221 (1993).

[4] J. Herbich, A. Kapturkiewicz, Chem. Phys. Lett. 273, 8 (1997).

[5] J. Dobkowski, J. Waluk, Polish J. Chem. 4, 1389 (1993).

[6] O. Anner, Y. Haas, J. Phys. Chem. 90, 4298 (1986).

[7] S. Cogan, S. Zilberg, Y. Haas, J. Am. Chem. Soc. 128, 3335 (2006).

[8] K.A. Zachariasse, Chem. Phys. Lett. 320, 8 (2000).

[9] K.A. Zachariasse, M. Grobys, Th. Van der Haar, A. Hebecker, Yu.V. Il'ichev, O. Morawski, I. Rücker, W.J. Kühnle, J. Photochem. Photobiol. A 105, 373 (1997).

[10] Z.R. Grabowski, K. Rotkiewicz, A. Siemiarczuk, D.J. Cowley, W. Baumann, Nouv. J. Chim. 3, 443 (1979).

[11] C. Rullière, Z.R. Grabowski, J. Dobkowski, Chem. Phys. Lett. 137, 408 (1987).

[12] P.B. Barbara, W. Jarzȩba, in: Advances in Photochemistry, Vol. 15, Eds. D.H. Volman, G.S. Hammond, K. Gollnick, Wiley 1990, p. 1.

[13] M.J. Horng, J.A. Gardecki, A. Papazyan, M. Maroncelli, J. Phys. Chem. 99, 17331 (1995).

[14] S.I. Druzhinin, V. Galievsky, T. Yoshihara, K.A. Zachariasse, J. Phys. Chem. A 110, 12760 (2006). 
[15] J. Dobkowski, Z.R. Grabowski, J. Waluk, W. Kühnle, W. Rettig, C. Rullière, W. Yang, J. Adamus, J. Gȩbicki, Proc. Indian Acad. Sci. (Chem. Sci.) 104, 143 (1992).

[16] (a) G.M. Kosolapoff, J. Am. Chem. Soc. 69, 1651 (1947). (b) R. Gawinecki, S. Andrzejak, A. Puchała, Org. Prep. Proced. Int. 30, 455 (1998).

[17] J. Jasny, J. Lumin. 17, 149 (1978).

[18] R.A. Velapoldi, in: National Bureau of Standards Special Publication 378, Accuracy in Spectrophotometry and Luminescence Measurements, Proc. Conf. NBS, Gaithersburg, MD, 1972, p. 231.

[19] J. Dobkowski, V.A. Galievsky, J. Jasny, I.V. Sazanovich, Polish J. Chem. 78, 961 (2004).

[20] T. Yoshihara, V.A. Galievsky, S.I. Druzhinin, S. Saha, K.A. Zachariasse, Photochem. Photobiol. Sci. 2, 342 (2003).

[21] W. Liptay, Z. Naturforsch. A 17, 621 (1962).

[22] W. Liptay, in: Excited States, Vol. 1, Ed. E.C. Lim, Academic Press, New York 1974, p. 129.

[23] M. Maus, W. Rettig, D. Bonafoux, R. Lapouyade, J. Phys. Chem. 103, 3388 (1999).

[24] T. Shida, in: Electronic Absorption Spectra of Radical Ions. Physical Science Data, Vol. 34, Elsevier, Amsterdam 1988, p. 275.

[25] J. Dobkowski, E. Kirkor-Kamińska, J. Koput, A. Siemiarczuk, J. Lumin. 27, 339 (1982).

[26] S.K. Lower, M.A. El-Sayed, Chem. Rev. 66, 199 (1966).

[27] S. Dähne, W. Freyer, K. Teuchner, J. Dobkowski, Z.R. Grabowski, J. Lumin. 22, 37 (1980).

[28] J. Lipkowski, A. Tabaszewska, private information.

[29] M. Maus, W. Rettig, Chem. Phys. 218, 151 (1997).

[30] J. Herbich, J. Waluk, Chem. Phys. 188, 247 (1994).

[31] J. Dobkowski, W. Rettig, J. Waluk, Phys. Chem. Chem. Phys. 4, 4334 (2002).

[32] J. Dobkowski, J. Waluk, W. Yang, C. Rullière, W. Rettig, New J. Chem. 21, 429 (1997).

[33] J.C.D. Brand, J. Mol. Spectrosc. 20, 359 (1966).

[34] C. Dubroca, Chem. Phys. Lett. 15, 207 (1972).

[35] C. Dubroca, P. Lozano, Chem. Phys. Lett. 24, 49 (1974). 